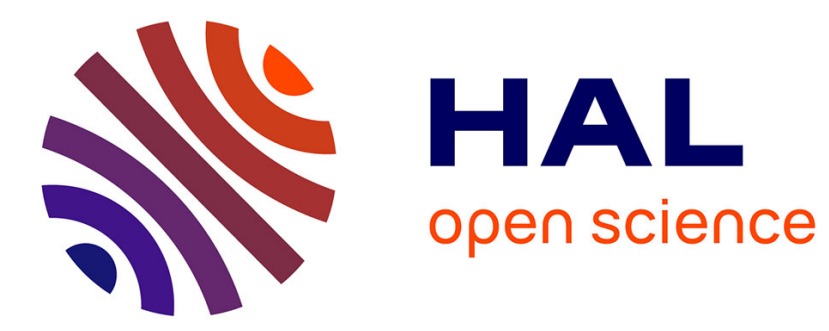

\title{
Adolescents, adolescentes face à la justice pénale
}

Arthur Vuattoux

\section{To cite this version:}

Arthur Vuattoux. Adolescents, adolescentes face à la justice pénale. Genèses. Sciences sociales et histoire, 2014, 97 (4), pp.47. 10.3917/gen.097.0047 . halshs-01849049

\section{HAL Id: halshs-01849049 \\ https://shs.hal.science/halshs-01849049}

Submitted on 17 May 2019

HAL is a multi-disciplinary open access archive for the deposit and dissemination of scientific research documents, whether they are published or not. The documents may come from teaching and research institutions in France or abroad, or from public or private research centers.
L'archive ouverte pluridisciplinaire HAL, est destinée au dépôt et à la diffusion de documents scientifiques de niveau recherche, publiés ou non, émanant des établissements d'enseignement et de recherche français ou étrangers, des laboratoires publics ou privés. 


\section{Adolescents, adolescentes face à la justice pénale}

Version non éditée de la référence suivante : Vuattoux Arthur, « Adolescents, adolescentes face à la justice pénale », Genèses, 2014/4 (n 97), p. 47-66. DOI : 10.3917/gen.097.0047.

Auteur :

Arthur Vuattoux

Université Paris 13, IRIS (EHESS, UP13, Inserm, CNRS),

74, rue Marcel Cachin

F-93017 Bobigny (France),

vuattoux@univ-paris13.fr

\section{Résumé :}

Cet article propose un cadre de compréhension et étaye des hypothèses quant au traitement différentiel des adolescents selon le genre dans la justice pénale. Après une présentation des statistiques publiques de la délinquance des mineurs et de ses réponses institutionnelles, analysées au prisme du genre, on examinera d'un point de vue qualitatif le travail judiciaire et ses productions (écrits éducatifs, dossiers judiciaires), afin de saisir, dans leur banalité, les biais de genre opérant quotidiennement dans le traitement social des « mineurs délinquants » par la justice. Cette analyse confrontée aux propos des professionnels (éducateurs et magistrats), recueillis au cours d'une enquête par observations et entretiens réalisée au Tribunal pour enfants de Créteil entre juin 2012 et juin 2013. L'enjeu consiste, dès lors, à décrire dans ses aspects les plus concrets la production et reproduction des normes de genre et ses effets sur les carrières institutionnelles des adolescents. 


\section{Adolescents, adolescentes face à la justice pénale}

Prises en charge dans le cadre de l'Ordonnance du 2 février 1945, les adolescentes délinquantes ${ }^{\mathrm{i}}$ font l'objet d'un traitement pénal qui révèle une sous-représentation des filles et donne lieu à diverses hypothèses. Souvent attribuée à des variations dans la socialisation des garçons et des filles, qui amèneraient ces dernières à des formes de déviance moins pénalisées, cette sous-représentation semble également dépendre de causes institutionnelles. Ainsi, afin de se départir d'une étiologie essentialiste ou d'une explication basée sur la seule socialisation, il est nécessaire de mettre au jour le sous-texte genré des réponses judiciaires : les filles font-elle l'objet d'un traitement différentiel par l'institution, et si c'est le cas, par quels mécanismes cette différence opère-t-elle?

L'enquête de terrain sur laquelle s'appuie cet article s'est déroulée entre juin 2012 et juin 2013. Quatre mois ont été consacrés à l'observation du quotidien d'un service éducatif,l'Unité éducative auprès du tribunal (UEAT), service de la Protection judiciaire de la jeunesse intégré au tribunal pour enfants, le reste du temps ayant été divisé en différentes périodes d'observations d'audiences et de travail sur les archives du tribunal (dossiers judiciaires des années 2010 à 2012, en assistance éducative et au pénal). Les données qualitatives dont sont issus cas présentés plus bas proviennent essentiellement de l'étude des rapports éducatifs produits par l'UEAT, mais des éléments tirés des dossiers judiciaires seront également utilisés en conclusion. Les observations réalisées au tribunal, ainsi que des entretiens (individuels et en focus group), permettront par ailleurs de comprendre le contexte de production de ces écrits et leur fonction dans la chaîne pénale. Souvent lus et annotés par les magistrats, parfois même discutés avec les éducateurs (il n'est pas rare qu'un juge confronte sa lecture du dossier à celle d'un éducateur de l'UEAT), ces écrits ont un rôle de cadrage de la situation du mineur. Mêlant indications « factuelles » et regard éducatif « subjectif », ils sont traversés par des formes implicites ou explicites de jugement sur ces situations, et il semble pertinent de les analyser à l'aune des normes de genre. Il s'agit, à travers les écrits discutés dans cet article, de saisir au plus près les processus de production et reproduction des normes de genre dans le travail des professionnels de la justice, avec pour objectif premier d'éclairer la production institutionnelle des décisions de justice au prisme du genre et de ses effets sociaux.

L'objectif de cet article consiste à mettre au jour le rôle des normes de genre dans le sentencing ${ }^{i i}$, c'est-à-dire dans la pratique sociale du jugement (manière de juger, cadres d'interprétation des professionnels de la justice, etc.). Pour ce faire, le niveau des interactions (ici essentiellement représenté par le compte-rendu de ces interactions et leur analyse qualitative) est aussi nécessaire à étudier que les dimensions quantitatives de l'activité judiciaire ou policière. Comme le notent Françoise Vanhamme et Kristel Beyens à propos de l'appréhension sociologique des discriminations à l'œuvre dans la justice,

« La discrimination se construit au niveau individuel des interactions à l'audience, dans des aspects subtils peu appréhendables par les statistiques, comme la conduite, l'argumentation et le vocabulaire respectifs, les perceptions et jugements implicites du 
style de vie, de l'avenir... Ce ne sont donc pas les caractéristiques des accusés qui peuvent en soi expliquer les discriminations, mais bien leur interprétation, en association avec l'évaluation du degré de faute et de dangerosité. C'est pourquoi la compréhension du sentencing a besoin surtout de recherches qualitatives et interprétatives favorisant la complexité » (Vanhamme, Beyens 2007 : 207-208).

Une première partie de l'article mobilisera des données issues des statistiques publiques (statistiques de la délinquance et de l'activité judiciaire). Aussi utiles soient-elles, ces données doivent être passées au crible de la critique : souvent vouées à justifier des politiques publiques, elles manquent parfois de précision ou d'une rigueur méthodologique autorisant leur usage par les sciences sociales. Elles permettent toutefois de préciser et de concrétiser l'idée de filtre de disparition des filles dans la chaîne pénale, en confirmant leur sous-représentation à toutes les étapes de cette chaîne.

Les données qualitatives de cette recherche, quant à elles, seront présentées dans une deuxième partie de l'article, à travers l'analyse des écrits éducatifs de l'UEAT. Ces écrits permettent de comprendre la manière dont les perceptions et attentes implicites des acteurs se déclinent selon le genre des justiciables. Les observations menées à l'UEAT, ainsi que les entretiens réalisés auprès des producteurs de ces écrits permettent par ailleurs d'éclairer leur inscription dans un ordre normatif (notamment en matière de normes de genre) qui dépasse le seul cadre professionnel. Ces matériaux donneront lieu à la formulation d'hypothèses concernant le poids des normes de genre dans la chaîne judiciaire.

Une dernière partie de l'article présentera les principaux axes de discussion élaborés à partir de ces hypothèses, en mobilisant notamment, à la marge, d'autres aspects des dossiers judiciaires (procèsverbaux policiers, rapports éducatifs, évaluations psychologiques, etc.).

\section{Quantifications : les enseignements d'une analyse genrée des statistiques publiques}

Avant d'évoquer les normes de genre et leur impact dans les écrits éducatifs au tribunal pour enfants, il paraît nécessaire de faire état des données disponibles en termes de sex ratio dans la justice des mineurs, et ce tout au long de la procédure pénale. Inégales du point de vue de la solidité statistique, ces données n'en demeurent pas moins susceptibles d'être analysées dans le but de révéler d'éventuels biais de genre dans la chaîne pénale ${ }^{\text {iii }}$. Il s'agit notamment de mettre au jour les différents filtres expliquant la disparition progressive des adolescentes au fil des procédures judiciaires.

Pourquoi parle-t-on de filtre de disparition des filles dans la chaîne pénale ? Cette notion, mobilisée par Coline Cardi au sujet des femmes déviantes (Cardi 2008 : 76), permet de mettre en évidence les biais institutionnels d'orientation des dossiers judiciaires et de choix des procédures en fonction du 
sexe des prévenus. L'idée de filtre de disparition renvoie notamment à l'usage différentiel des procédures d'abandon de poursuite, de classement ou de relaxe, ainsi qu'à la non-transmission des procédures au Parquet lorsqu'il s'agit d'adolescentes. Elle renvoie également, de manière encore plus difficile à mesurer, à la pratique consistant à transférer au civil (assistance éducative) la charge de traiter une situation qui relève pourtant du pénal au regard des infractions commises. Enfin, il est probable que le recours à des prises en charge non-judiciaires (prises en charge locales par les services sociaux ou scolaires, prises en charge médico-psychologiques et hospitalisations en psychiatrie) explique en partie la réorientation des dossiers d'adolescentes hors de la chaîne pénale. Nicolas Herpin insistait, dans ses recherches sur les rapports de classe et la justice, sur l'influence des filtres de repérage des infractions quant à la «mutation » entre primo-délinquance et inscription d'un individu dans la délinquance de longue durée :

«Le tribunal ne produit pas à lui seul cette mutation. Sa décision a été préparée par celle du Parquet et de l'instruction. Il faudrait remonter plus en amont encore, à l'activité des polices, à la surveillance des voisinages, pour reconstituer la formation du phénomène criminel. L'emboîtement successif de ces filtres, s'il pose des problèmes considérables pour l'analyse empirique, a le mérite de faire apparaître la criminalité comme un produit social. Le délinquant n'est pas d'abord celui qui "passe à l'acte", comme le représentent certains penseurs officiels de la criminologie française. Il ne suffit pas, en effet, de commettre un acte répréhensible pour être repéré et, a fortiori, condamné. L'infraction ne fait pas le larron » (Herpin 1977 : 132).

C'est à la mise en évidence de tels filtres, cette fois selon le genre, que sert une lecture critique des statistiques publiques produites à propos des mineurs dans la chaîne pénale.

On distingue généralement trois phases principales dans la chaîne pénale : l'amont de l'intervention judiciaire (avant la transmission des informations au Parquet de secteur par la Police ou la Gendarmerie), l'intervention judiciaire en tant que telle (qualification juridique, investigation, instruction, audiences et décisions de justice), et enfin son aval (l'application des décisions de justice, par exemple la détention, le suivi éducatif en milieu ouvert ou dans le cadre d'un placement). Afin de mesurer l'importance quantitative de la délinquance des filles et d'en comprendre les enjeux genrés ${ }^{\text {iv }}$, il convient de s'intéresser à l'ensemble de ces étapes, en se focalisant sur les données de sex ratio à chaque niveau d'intervention des institutions concernées (Police, Justice, Protection judiciaire de la jeunesse, etc.), et en prenant pour indicateur le pourcentage d'adolescentes parmi l'ensemble des mineurs concernés (filles et garçons). Il est par ailleurs nécessaire d'être conscient des limites propres aux statistiques fournies par les institutions, tant du point de vue de leurs méthodologies que de leur comparabilité, la fiabilité des informations produites par la Police sur les « mis en cause », basées sur l'exploration de données directement codées par les policiers en fonction de leur activité, n'étant par exemple pas similaire à celle des condamnations inscrites au Casier judiciaire national et rendues publiques par le ministère de la Justice, correspondant à l'activité exhaustive de l'institution. 
Cependant, ces données demeurent riches en informations quant au traitement différentiel des mineurs selon le genre

Considérons l'amont de la chaîne pénale, c'est-à-dire l'étape des mises en cause par la Police et la Gendarmerie. Catégorie administrative, la notion de «mis en cause» désigne des personnes faisant l'objet d'une procédure en tant qu'auteurs. Les statistiques policières sont établies à partir d'un ensemble de données appelé « état 4001 », du nom de l'outil employé par les services de Police et de Gendarmerie et servant de base à la présentation annuelle des données de la délinquance par l'Observatoire national de la délinquance et des réponses pénales (ONDRP) ${ }^{\mathrm{v}}$. Les données obtenues via l'état 4001 sont très discutables si l'on veut y voir le reflet fidèle de la délinquance; en revanche, malgré des doutes subsistants sur le bon usage de cet outil par les policiers, elles renseignent assez bien l'activité policière,. Si l'on s'intéresse aux seuls mineurs, à partir des données fournies, pour l'année 2011, par l'ONDRP (Scherr, Perron-Bailly, 2012) la proportion des «femmes mineures » parmi l'ensemble des mineurs mis en cause s'établit à $17,2 \%$ en $2011^{\text {vi }}$, avec une progression importante des mises en cause chez celles-ci entre 2006 et $2011(+24,4 \%$, contre une baisse de 2,9\% des mises en cause de garçons durant la même période). Ces données, plus que toutes les autres, doivent être considérées avec précaution. Comme le note Bruno Aubusson de Cavarlay, l'état 4001 exclut un grand nombre d'infractions légères (procédures policières ou de gendarmerie ne faisant pas l'objet d'une transmission au Parquet, ou faisant l'objet de simples contraventions), dont on sait par ailleurs qu'elles sont caractéristiques des délits commis par les mineurs (Aubusson de Cavarlay 2013 : 1). De plus, l'usage des statistiques policières dans le débat politique entretient une tendance sécuritaire, conduisant les ministres de l'Intérieur successifs à organiser une mise en cause accrue des mineurs délinquants via un renforcement de l'activité policière. Enfin, une autre hypothèse peut être avancée, à partir du constat de l'augmentation des mises en cause féminines : cette hypothèse consiste à imputer cette hausse à une transformation de la représentation sociale des délinquants, et en l'occurrence des délinquantes, dont le pouvoir de nuisance serait devenu plus perceptible. Ainsi, les services de sécurité des commerçants ont-ils sans doute davantage signalé de vols commis par des femmes dans les dernières années, du fait d'un repérage plus proactif de ces dernières et notamment d'une sensibilisation accrue à une forme de délinquance présentée comme nouvelle (Ocqueteau 2006 : $35)$.

Il convient maintenant de se pencher sur les suites données à ces mises en cause par les services de Police et de Gendarmerie, en rendant compte de l'activité des tribunaux telle que les chiffres fournis par le ministère de la Justice les donnent à voir. Ces données sont peu exploitables dans le cadre d'une approche par le genre, car elles n'ont que très récemment intégré la variable « sexe ». On peut toutefois extraire de ces statistiques l'information suivante, qui peut être mise en lien avec les données de sex ratio parmi les «mis en cause » : la part des filles dans les situations pour lesquelles un juge des enfants a été saisi s'établissait en 2011 à 10,1\% (proportion stable entre 2009 et 2012, puisque comprise entre 9,5 et $10,1 \%$ sur cette période) $)^{\mathrm{vii}}$. 
Il serait intéressant, concernant les statistiques pénales, de connaître le sex ratio en fonction de l'orientation donnée aux affaires par le Parquet, et notamment de connaître la part des adolescentes dans les procédures alternatives aux poursuites. Ces statistiques n'existent malheureusement pas à l'heure actuelle, et on doit donc leur substituer des données recueillies localement. Ainsi, l'enquête ethnographique a permis d'observer, à partir de quelques affaires, les enjeux genrés du recours à de telles procédures ${ }^{\text {viii. }}$. Il serait particulièrement heuristique de pouvoir renseigner la part des adolescentes dans les «compositions pénales », mesures dites de troisième voie permettant au procureur de proposer des mesures à dimension éducative ou des amendes sans enclencher la procédure judiciaire habituelle (dans les compositions pénales, le juge ne fait que valider la procédure négociée en amont par le Parquet et le service éducatif). Il est fort probable que des enjeux de genre se révèlent dans ces procédures : on peut à cet égard faire l'hypothèse selon laquelle les compositions pénales sont privilégiées chez les adolescentes, dont la délinquance reste une exception dans les représentations sociales, par opposition à des garçons pour lesquels les procédures pénales « classiques » (mesures et sanctions éducatives notamment) ont été initialement pensées.

Enfin, à l'autre extrémité de la chaîne pénale, on peut mobiliser les données issues du Casier judiciaire national, organe de comptage et d'enregistrement des condamnations signalées par les différentes juridictions; ces données sont rendues publiques chaque année par le ministère de la Justice (Ministère de la Justice 2013 : 227-228). Si l'on se réfère aux données de l'année 2011, on observe un ratio d'environ une fille pour neuf garçons dans les condamnations (incluant les peines privatives de liberté, les mesures éducatives et les dispenses de peine). Ainsi, les filles représentent 9,5\% des condamnations principales de mineurs enregistrées au Casier judiciaire national. Si l'on décompose par type de condamnation, elles représentent seulement $7 \%$ des peines privatives de liberté, $11,8 \%$ des mesures et sanctions éducatives et $13 \%$ des dispenses de peine. On remarque, dès lors, que la part des filles est plus importante dans les mesures éducatives ou les dispenses de peines, ce qui indique qu'elles connaissent, du strict point de vue de la gravité des peines, une issue plus favorable dans les procédures judiciaires qui les concernent.

Le schéma suivant permet de situer les données connues en matière de sex ratio sur l'axe de la chaîne pénale, en indiquant la part des filles parmi l'ensemble des mineurs (filles et garçons) à diverses étapes du processus judiciaire : 


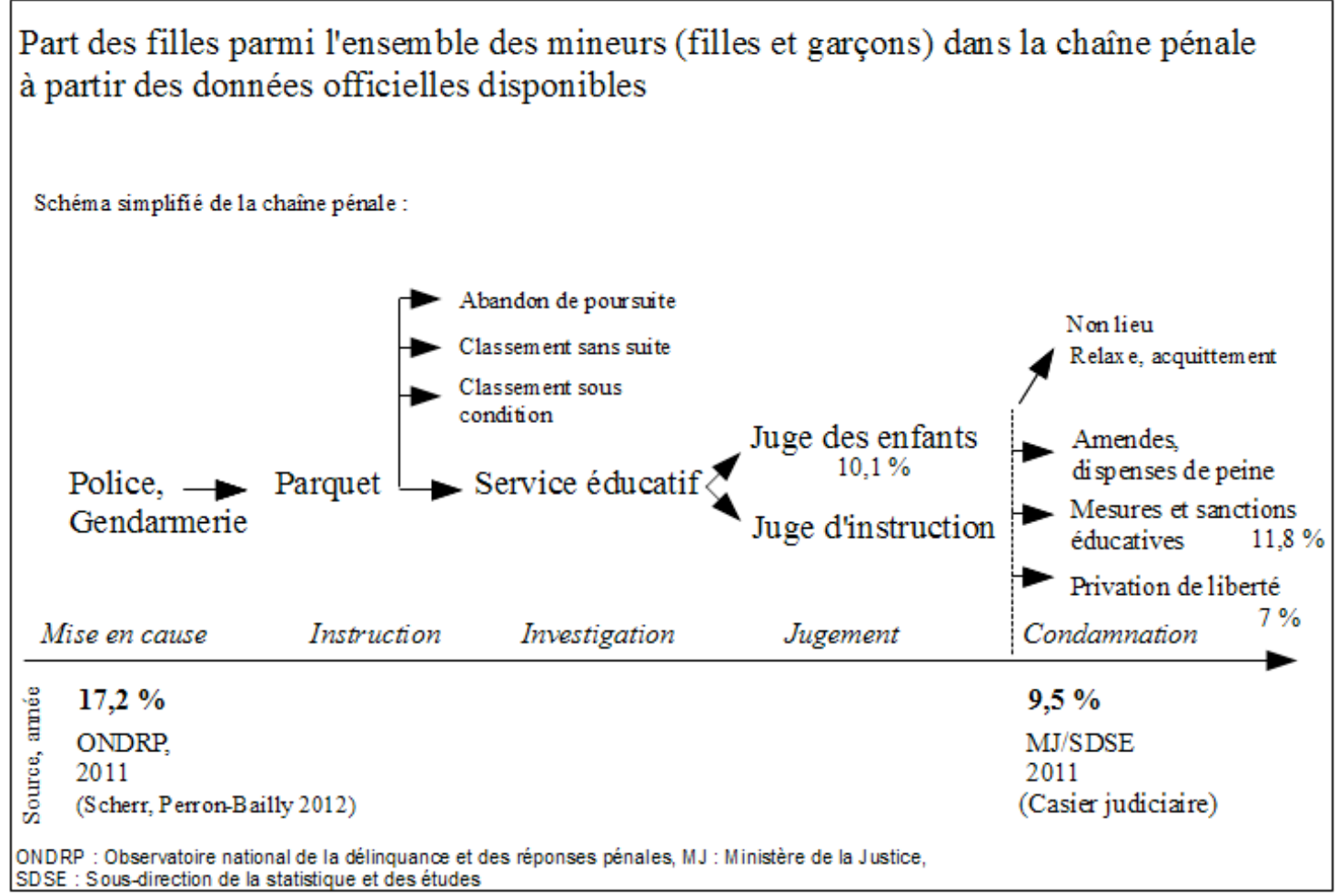

Ce schéma simplifié permet de conforter l'hypothèse selon laquelle les adolescentes voient, dans le cours la chaîne pénale, leurs dossiers orientés vers des prises en charge qui ne sont pas similaires à celles des garçons, d'où l'idée d'un filtre de disparition des filles dans la chaîne pénale, corrélé à une réorientation des dossiers de mineures vers des prises en charge para-pénales. Cependant, il convient de rappeler les limites de ces données en termes de fiabilité statistique et de comparabilité. Il est, en la matière, mal aisé de distinguer les effets de genre produits par l'institution d'effets liés, en amont de toute intervention judiciaire, à la socialisation des filles et des garçons, les amenant à commettre des délits différents et conduisant, ainsi, à des réponses institutionnelles différenciées.

À ce stade de l'analyse, on repère les limites d'une approche qui s'appuierait sur les seules données quantitatives existantes. C'est pourquoi je propose, dans les lignes qui suivent, de rendre compte d'une approche qualitative de la chaîne pénale et de ses filtres genrés. La nécessité d'un niveau qualitatif dans ce type d'analyse, mentionnée plus haut, incite à recueillir différents ordres de données : les dossiers judiciaires et, plus spécifiquement, les écrits issus de la phase d'investigation éducative au tribunal, les propos des acteurs de la chaîne pénale (et notamment ceux des éducateurs et magistrats) et, enfin, certains résultats issus de l'observation au long cours du tribunal et de son unité éducative. 


\section{Le filtre du genre à l'épreuve de l'empirie : une enquête auprès des acteurs de l'investigation éducative}

Lors de l'enquête ethnographique réalisée au tribunal pour enfants de Créteil, divers aspects de l'activité judiciaire ont été étudiés, via des observations (observation d'audiences de cabinet et d'audiences du Tribunal pour enfants, observation du quotidien de l'Unité éducative auprès du tribunal), des entretiens (entretiens individuels avec des magistrats, éducateurs et acteurs auxiliaires de la justice des mineurs; entretiens collectifs sous forme de focus groups $^{i x}$ ), et la lecture de dossiers judiciaires et, plus spécifiquement, des écrits éducatifs de l'UEAT : les RRSE, recueils de renseignements socio-éducatifs, composante importante des dossiers judiciaires pénaux. Les matériaux mobilisés ici sont principalement issus de la partie du terrain menée à l'UEAT, d'une part afin de concentrer l'analyse sur des matériaux bien délimités (les écrits éducatifs et leurs usages), d'autre part du fait de l'intérêt que présente la phase d'investigation éducative dans une analyse des biais propres à la chaîne pénale. Comme l'écrit Bernard Cassagnabère, L'UEAT est « une véritable plaque tournante de la prise en charge des mineurs délinquants et un formidable outil d'observation des évolutions tant du phénomène de la délinquance des mineurs que des réponses judiciaires » (Cassagnabère 1996 : 160).

Évoquons en premier lieu le rôle de l'UEAT et la fonction des acteurs qui y travaillent au sein de la chaîne pénale. D'un point de vue institutionnel et légal, l'UEAT dépend du service territorial éducatif de milieu ouvert (STEMO), son existence date de 1987. La nécessité de l'investigation éducative est inscrite dans le texte de référence de la justice pénale des mineurs - l'Ordonnance du 2 février 1945 qui précise dans son article 12 que lors des défèrements de mineurs au tribunal, "le service de la protection judiciaire de la jeunesse compétent établit, à la demande du procureur de la République, du juge des enfants ou de la juridiction d'instruction, un rapport écrit contenant tous renseignements utiles sur la situation du mineur ainsi qu'une proposition éducative $»^{\mathrm{x}}$. Une circulaire du 31 décembre $2010^{\mathrm{xi}}$ détaille le contenu de ce document, qui doit porter essentiellement sur la situation matérielle et morale du jeune et de sa famille, la personnalité du mineur, son rapport à l'école, son développement psychologique ou encore sa santé.

Parmi les outils à la disposition des éducateurs pour effectuer leurs investigations dans le cadre pénal, je m'intéresserai ici aux RRSE, éléments centraux dans l'activité quotidienne du service car correspondant à la retranscription du travail d'investigation éducative mené dans le service, transmis ensuite au juge des enfants. Les RRSE sont donc des comptes-rendus écrits de la rencontre entre un éducateur et un jeune déféré, ainsi qu'avec sa famille lorsqu'elle est présente au tribunal. Rédigés par les éducateurs au cœur de la phase d'investigation au tribunal, ils agissent à la fois comme un outil de contrôle (vérification de l'identité du jeune, de son contexte familial, éducatif, de son environnement social) et comme la base matérielle de transmission des informations entre le Parquet, les éducateurs et les juges des enfants. Seule trace matérielle de la phase d'investigation versée au dossier judiciaire, les 
RRSE documentent le poids des normes de genre au sein de la chaîne pénale, à un niveau local et dans un segment limité de l'activité judiciaire - celui des affaires de délinquance transmises aux juges des enfants et faisant l'objet de la procédure la plus classique.

La rencontre au dépôt, point de départ de ces écrits, est un élément important du point de vue des professionnels, qui considèrent parfois cette rencontre comme un moyen de «jauger» la gravité de la situation. Certains m'ont expliqué, par exemple, qu'il existait une grande différence entre ce que laisse supposer le délit commis, le choix du Parquet (c'est-à-dire la poursuite), et leur rencontre avec des mineurs parfois effondrés par la situation et laissant échapper, lors de l'entretien avec les éducateurs, des informations capitales telles qu'une situation familiale délétère, ou leur inscription dans un réseau de criminalité dont ils se disaient victimes. La présente recherche, focalisée sur les professionnels, ne permet pas de rendre compte du point de vue des mineurs. Cependant, il semble, d'après les quelques observations réalisées au dépôt, que certains mineurs (ceux qui ont une expérience de l'institution judiciaire) peuvent envisager cet entretien sous un angle stratégique, en faisant en sorte que les éducateurs aboutissent à une recommandation favorable, quand d'autres profitent de ce moment pour révéler des éléments biographiques qu'ils n'étaient pas à même de confier aux policiers lors de leur garde-à-vue. Dans les interactions au dépôt, les éducateurs paraissent souvent ambivalents, entre une confiance a priori accordée à la parole des jeunes et une mise à l'épreuve de cette parole au cours de l'interaction (à travers des demandes d'éclaircissement ou la mise au jour d'incohérences dans le récit des jeunes déférés).

Les RRSE ont un impact particulier dans les dossiers judiciaires du fait de leurs circulations : ils sont écrits par des éducateurs et éducatrices, lus et pris en considération par les juges des enfants, puis comparés à d'autres documents visant à établir la personnalité des adolescents. Placés dans une injonction paradoxale, entre mission éducative et nécessité de livrer au juge des éléments factuels sur lesquels se basera son pouvoir de sanction, les éducateurs de l'UEAT ne peuvent se cantonner à un avis strictement éducatif et doivent anticiper et proposer d'éventuelles sanctions. S'ils ne le font pas, le risque est grand de voir les juges se désintéresser de leurs écrits. En effet, si le travail des éducateurs de l'UEAT s'avère relativement indépendant des injonctions du Parquet, il s'effectue en revanche en lien étroit avec le travail des juges des enfants. Les RRSE sont en théorie rédigés selon une trame proposée aux UEAT par l'administration centrale de la Protection judiciaire de la jeunesse. Dans les faits, les éducateurs, comme certains l'ont confié lors de l'enquête, ne s'y réfèrent que vaguement : la trame leur sert de point de repère concernant les thèmes à aborder, mais n'est jamais totalement renseignée. D'après cette trame, par exemple, il faudrait systématiquement renseigner sur le détail du parcours scolaire des jeunes, ou sur leur éventuelle expérience migratoire. Or, comme certains éducateurs me l'ont indiqué, il est difficile, dans le temps imparti pour la rédaction de ce rapport d'obtenir suffisamment d'éléments. De plus, les éducateurs se forment généralement sur le tard à la rédaction de ces rapports, leur formation à l'Ecole nationale de la Protection judiciaire de la jeunesse (ENPJJ) ne les préparant pas spécifiquement à ce type d'écrit. 
Concrètement, les RRSE consistent en un double feuillet A4, comprenant une première page composée de rubriques - état civil, taille de la fratrie, délit, nom du juge en charge du dossier, historique des passages au tribunal s'il y a lieu - ; c'est la partie la plus formalisée de ces écrits. Les autres pages ne comportent que de grands intitulés du type : entretien avec la famille (une page), entretien au dépôt (une page), observations et recommandations (une page).

La dernière partie des RRSE, leur conclusion, consiste donc en des « recommandations » faites au juge des enfants. Il s'agit de proposer un type de mesure, la relaxe, ou encore le recours à des formes de prises en charge para-pénales. Les éducateurs, au terme de leur brève rencontre avec un mineur déféré (l'entretien au dépôt excédant rarement la demi-heure) doivent donc faire un choix, dont l'enjeu est qu'il bénéficie au mineur et surtout qu'il soit suivi par le juge des enfants. Ces choix ne sont pas encadrés par des règles strictes, qui dictent qu'à situation $\mathrm{X}$ devrait être proposée la solution éducative Y. Les règles qui sont appliquées relèvent bien plutôt d'une culture éducative implicite, mais aussi d'une forme de négociation avec les juges. Par exemple, il semble admis, bien que cela soit implicite, que certaines réponses pénales sont à éviter dans l'absolu. La détention, y compris en centre éducatif fermé, ou encore les placements éloignés de la famille des adolescents ne sont jamais proposés par les éducateurs et on peut lire, au détour d'un rapport d'activité du service, qu'un taux d'incarcération de $10 \%$ est « trop élevé », indiquant le rejet de ce type de réponse pénale. De plus, la part de négociation existant entre les professionnels de l'UEAT et les magistrats s'actualise à travers un accord tacite sur certains principes, principes parfois discutés de visu, dans le bureau du juge. Par exemple, si le service éducatif parvient à prouver qu'un adolescent poursuivi pour une infraction lourde (donnant lieu à une peine de privation de liberté) est par ailleurs, depuis qu'il a commis ces actes, inscrit dans une forme de réinsertion jugée solide (reprise de scolarité, de formation ou emploi), le magistrat aura tendance à faire preuve de mansuétude. Mais là encore, rien d'automatique : l'enjeu est alors de savoir, dans chaque situation traitée à l'UEAT, si la décision prise par le magistrat sera bien en accord avec les recommandations éducatives. Ainsi, durant mon enquête, j'ai entendu à plusieurs reprises des éducateurs expliquer que tel juge risque de «ne pas suivre» car il a l'habitude de ne pas aller systématiquement dans le sens des recommandations du service, alors que d'autres le font plus volontiers. On voit ainsi la dimension stratégique que peut revêtir l'écriture des RRSE, du moins en ce qui concerne les recommandations des éducateurs qui concluent ces écrits.

La rédaction des RRSE s'effectue individuellement : un éducateur est chargé d'un dossier, qu'il suit du début (la rencontre avec le jeune au tribunal) jusqu'à la fin du processus d'investigation (la remise du rapport au juge, puis la consignation, dans un cahier de suivi à l'UEAT, de la décision prise par ce dernier). Pour les rédiger, chaque éducateur possède un bureau à l'UEAT, avec un ordinateur et, objet central, un téléphone lui permettant de contacter divers services éducatifs en mesure d'accueillir le jeune si le juge « suit » la recommandation éducative. En effet, l'une des fonctions du travail effectué à l'UEAT est de rendre les recommandations applicables : si le juge va dans le sens des éducateurs et décide, par exemple, d'un placement en foyer, les éducateurs doivent pouvoir garantir qu'un foyer 
accueillera bien le jeune dès que la décision prendra effet. Enfin, une autre dimension remarquable du travail des éducateurs est leur rôle dans la constitution d'un historique des défèrements de chaque mineur. Il est courant qu'un mineur soit présenté au juge pour la deuxième ou la troisième fois, et les éducateurs doivent, dans ce cas, retrouver les RRSE précédemment établis et les mobiliser dans leur analyse (par exemple, en mettant au jour une difficulté familiale qui était apparue lors d'un précédent défèrement, et dont le juge pourra prendre connaissance pour l'intégrer à sa réflexion).

Ces écrits révèlent-ils des biais de genre susceptibles de venir en appui de l'hypothèse selon laquelle l'institution judiciaire tend à reproduire des normes dans son travail quotidien ? C'est pour répondre à cette question que l'enquête ethnographique a été focalisée, durant plusieurs mois, sur le travail des éducateurs à l'UEAT. Dans l'espace de cet article, il est possible de poser quelques jalons pour une analyse genrée du travail éducatif au tribunal.

Tout d'abord, signalons que la recherche menée à l'UEAT, présentée aux acteurs pour ce qu'elle est réellement - c'est-à-dire une recherche sur le genre et son impact sur le traitement pénal des mineurs -, a d'emblée suscité une forte adhésion de la part des professionnels. La profession d'éducateur à la PJJ est fortement féminisée, et l'UEAT de Créteil en constitue un exemple : 4 des 6 éducateurs sont en effet des éducatrices, même si, conformément à la réalité d'un "plafond de verre " ${ }^{\text {xii }}$ dans la fonction publique, les deux cadres éducatifs (directeur et responsable d'unité éducative) sont des hommes. De leur côté, les éducatrices rencontrées manifestèrent très rapidement un ethos féministe, ou tout au moins une sensibilité à la question du genre, repérable à leurs questionnements et à la manière de m'alerter, au cours de l'enquête, sur des situations qui leur paraissaient répondre à ma problématique. En voici un exemple, tiré de mon journal de terrain à l'UEAT :

« J'arrive vers $13 \mathrm{~h}$ à l'UEAT, afin de passer l'après-midi à travailler sur les écrits éducatifs récemment archivés. Les éducateurs et éducatrices de l'UEAT finissent leur déjeuner, et me proposent de boire un café à leur table, ce que j'accepte. Anne ${ }^{\text {xiii }}$, une éducatrice du service, commence alors à évoquer la situation d'une jeune fille dont elle dit qu'elle pourrait "m'intéresser". Il s'agit de la situation d'une adolescente de milieu aisé (son père étant "vétérinaire"), dont l'éducatrice dit avoir été frappée du fait du traitement favorable qui lui était réservé par les policiers, lié selon elle à l'appartenance de genre de l'adolescente. Elle m'explique la situation ainsi : "J'ai été frappée : quand je suis arrivée au dépôt, la jeune fille, une petite bombe, une ado très jolie, était hors cellule, sans menotte, ce qui n'arrive jamais... En plus, j'ai appris que pendant sa garde à vue de $24 \mathrm{~h}$, elle a eu droit à une douche, je n'avais jamais vu ça... Elle a eu un traitement de faveur, elle venait de Martinique et dans ces affaires, normalement, la première chose prononcée est une interdiction de sortie du territoire [métropole]. Là, le père a envoyé une longue lettre, et on était même prêt à lui payer un billet de retour !". Son collègue Amin réagit, affirmant que la jeune fille devait etre "sacrément jolie" pour que les policiers agissent ainsi. La discussion se poursuit ensuite afin de savoir si c'est le genre de cette jeune fille ou son milieu social qui a joué dans cette configuration inhabituelle. Certains des éducateurs présents penchent pour l'une ou l'autre des explications, ou pour la conjonction de ces deux éléments (...)» (extrait du journal de terrain, UEAT de Créteil, 18 janvier 2013). 
Outre le fait qu'il révèle une forte réflexivité des éducateurs de l'UEAT en matière de rapports de genre et de rapports de classe, cet extrait permet d'illustrer l'un des questionnements concernant les biais de genre dans la chaîne pénale, à travers l'idée que les filles seraient davantage favorisées que les garçons. Par hasard, j'ai pu retrouver, dans les archives consultées, le dossier de la jeune fille en question, à propos de laquelle il est effectivement mal aisé de distinguer l'influence respective du genre et de la classe dans les suites données à son délit. Dans le RRSE rédigé à son propos, on ne compte que quelques lignes consacrées au délit commis, placé au second plan des préoccupations éducatives (à l'instar de nombre des écrits étudiés concernant des adolescentes). En revanche, un long paragraphe s'attarde sur les difficultés psychologiques de l'adolescente, et notamment son rapport à l'école, qu'elle dit vivre comme une "prison » (les termes qu'elle emploie sont cités dans le RRSE). Il est écrit que son père semble imposer à la jeune fille des attentes démesurées, et que cette pression la conduit à un rejet de l'autorité familiale, rejet pouvant expliquer son inscription récente dans une conduite délinquante. Enfin, les mots employés dans la recommandation finale laissent planer un doute sur l'interprétation de ces éducateurs, en laissant apparaître une éventuelle prise en compte de son statut «particulier» (en tant que fille délinquante $o u$ en tant que fille délinquante issue d'une classe sociale favorisée). L'éducatrice rédige en effet ainsi sa recommandation : «En raison de sa personnalité et de la situation, un foyer PJJ ne paraît absolument pas adapté ».

D'autres écrits étudiés conforteront l'idée (valable pour les filles comme pour les garçons, mais selon des modalités différentielles), d'une grille de lecture présidant à la rédaction des écrits éducatifs. Comme dans le cas de cette jeune fille, il est rare de voir les éducateurs s'arrêter longuement sur les délits commis, alors que ceux-ci semblent déterminants dans l'analyse des situations des garçons. D'une manière générale, il apparaît que le regard des éducateurs, concernant les adolescentes, se porte davantage sur les difficultés intimes ou « intérieures » de leur parcours (sur leurs « blessures intimes », leur « intériorité » ou autres éléments renvoyant à une difficulté psychique supposée), alors que les adolescents sont davantage questionnés sur leurs difficultés sociales ou scolaires (on questionne davantage leurs « fréquentations », d'éventuelles « mauvaises rencontres » ou des habitudes supposées « antisociales » : consommation d'alcool, « occupation » outrancière de lieux publics).

Par exemple, voici ce que l'on peut lire dans un RRSE rédigé à propos d'un adolescent présenté au juge pour «Infraction à la législation sur les stupéfiants » (catégorie policière et pénale pouvant recouvrir une large gamme d'infractions liées aux stupéfiants, de l'usage à la possession, voire à la revente) :

«[Partie du RRSE consacré à la rencontre avec les parents:] Durant l'essentiel de l'entretien, les parents nous confirment les propos de leur fils concernant les rythmes, les règles et les relations de chacun. Nous comprenons à cette occasion que c'est Monsieur qui s'est beaucoup battu pour que Nassim obtienne l'orientation qu'il souhaitait. D'abord affecté en pâtisserie, Monsieur a saisi l'inspection académique, a fait les démarches, relancé, et finalement, Nassim a pu intégrer la filière de son choix $(\ldots)$ »

On remarquera généralement, dans les écrits consacrés aux jeunes filles, une mise au second plan de l'école, sauf si elle paraît être la cause de difficultés intimes révélées lors de l'entretien éducatif. En 
revanche, on assistera, bien plus souvent, à une mise en évidence de difficultés psychologiques assez rarement mises en lumière dans le cas des garçons. Le cas de Lara, une jeune fille poursuivie pour vol, est exemplaire de ce point de vue. De manière assez classique, le rapport fait état de son " insolence » au dépôt, avant d'en venir à des dimensions présentées comme plus "profondes » de sa situation, faisant intervenir son psychisme tout autant que son corps ou ses relations extra-familiales :

« La mère de Lara réclame le placement de sa fille, tout en ajoutant qu'elle souhaite aussi, bien-sûr, la protéger. Madame craint que Lara se prostitue et/ou qu'elle vende de la drogue. Elle se plaint que des adultes viennent constamment la chercher dans de grosses voitures, considérant qu'il s'agit automatiquement de dealers ou de proxénètes $(\ldots)$ ».

Et de conclure :

«Lara est une adolescente en très grande souffrance. Elle fuit toute forme de quotidienneté ou d'attache familiale et personnelle. Elle met constamment en avant son envie de liberté, de vivre au jour le jour sans contrainte... Elle semble fuir la réalité et les difficultés liées à une vie en société ».

De même, le RRSE de Aïcha, pour qui les mots "inquiétude » ou "difficulté » apparaissent à de nombreuses reprises ou celui de Mona, une jeune mineure étrangère (italienne) poursuivie pour vol, et dont le RRSE mentionne à plusieurs reprises les traits fatigués. Fait étonnant à ce propos : le corps ou les attitudes corporelles des garçons ne sont que très rarement décrits dans les RRSE, alors que les écrits qui concernent des jeunes filles sont davantage incarnés.

Ces quelques éléments, sans prétendre dessiner de manière exhaustive l'espace des représentations et stéréotypes véhiculés par les professionnels, permettent de donner une idée du type de biais d'appréhension des situations que l'on peut s'attendre à voir à l'œuvre dans le travail judiciaire. Si certains éléments semblent peu déterminants dans la réalisation de ces écrits, comme le sexe de l'éducateur rédigeant le RRSE, d'autres semblent jouer à plein, tels que le rapport de ces éducateurs aux normes dominantes en matière de genre, à travers, par exemple, la nécessité pour l'institution de «protéger» les filles lorsqu'il s'agit de «mettre un coup d'arrêt» plus ferme aux déviances des garçons (pour qui le cadre pénal semble d'emblée plus approprié).

C'est afin de pallier les limites des écrits éducatifs lorsqu'il s'est agi d'en interpréter les tenants et aboutissants, que des entretiens ont été réalisés avec les acteurs directement impliqués dans la production de ces écrits. Confronter ces écrits à leurs producteurs, notamment via des focus groups, a permis d'éclairer davantage l'hypothèse d'un traitement différentiel des adolescents selon leur genre. Les deux focus groups réalisés se déroulèrent à la fin de l'enquête de terrain, à un moment où je pouvais présenter et discuter des résultats préliminaires de mon enquête

Lors d'un focus group, la question de l'intimité a été posée sous l'angle de la difficulté qu'ont les éducateurs à l'aborder :

«Une éducatrice : Moi je pense qu'on ne peut pas mettre sur le même plan l'intervention de l'éducateur ou de l'éducatrice de l'UEAT, c'est-à-dire un seul entretien, et le travail d'accompagnement dans la durée où là, on a du temps, des moyens, des cadres pour 
aborder la question de l'intimité et de la sexualité... là on est dans le cadre d'un seul entretien...

Un cadre éducatif: Oui, mais en même temps, on observe des différences [dans le traitement de l'intimité entre filles et garçons] y compris dans les RRSE...

L'éducatrice : Oui mais le RRSE, c'est un instant $t$, un entretien. Pour moi, c'est pas la même chose... La question de l'intimité et de la sexualité n'est pas du tout quelque chose que je laisse de côté, ni avec les garçons, ni avec les filles (...). Je trouve que dans tous les cas, c'est des questions difficiles à aborder parce que peut-être qu'on ressent qu'on est intrusif, encore plus quand on parle de l'intimité ou de la sexualité que quand on parle de la scolarité (...) » (Focus group, UEAT, 2013).

Lors des focus groups, j'ai pu constater la variabilité des positions défendues par les professionnels de l'UEAT à propos du genre et de ses effets sur leurs écrits. Si la position la plus courante consistait à admettre un traitement différentiel, tout en mettant en évidence une étiologie différentielle des déviances (selon laquelle les filles ne présentent pas tout à fait le même profil que les garçons, expliquant en partie la différence de réponse institutionnelle qui leur est apportée), d'autres professionnels défendaient soit la seule influence de l'expression de genre des filles (au sens où les adolescentes mettraient elles-mêmes en avant leur intimité, «forçant» ainsi les professionnels à l'investiguer, contrairement aux garçons), soit l'absence totale de distinction entre filles et garçons dans leur travail.

Du côté des juges, les positions défendues sont globalement identiques, même si l'on observe, de leur part, une tendance à minorer leur rôle dans la production des normes, certains expliquent même «corriger», dans l'exercice de leur fonction, les dimensions trop fortement normatives des écrits éducatifs, à l'instar de la juge des enfants Michelle Plessard qui, au cours d'un entretien, attribuait la reproduction des normes de genre par les éducateurs à la prégnance des stéréotypes dans la société :

«On fait plus de reproches à une fille sur la manière dont elle se maquille, sur la manière dont elle sort... Et pourtant, moi je dis aux éducateurs, "elles ne sortent pas toutes seules, il y a des garçons avec elles !". De leur part, il n'y a pas forcément de reproche jugeant, mais de l'inquiétude. L'inquiétude elle va sur les filles, elle va moins sur les garçons qui, pourtant, sortent avec les filles... Je pense que c'est quand même le reflet de l'idée qu'on a des filles, de la protection, du fait que les garçons, ça ne parle pas trop d'eux, les filles plus facilement... Je pense que ça a un rapport (...). On pourrait dire que les écrits éducatifs sont le reflet de ce qu'attendent les juges, mais bon, on ne leur dicte pas leurs écrits... C'est aussi le reflet de ce qu'est la société. C'est vrai que dans le domaine des inquiétudes, on s'inquiète plus de l'apparence de la fille, de ce qu'elle peut faire la nuit, et on n'interroge pas les garçons qui peuvent faire la même chose... » (Entretien avec Michelle Plessard, juge des enfants, Créteil, juin 2013).

Ces matériaux, qu'il s'agisse d'écrits éducatifs, d'observations réalisées au tribunal ou d'entretiens avec les acteurs de la justice des mineurs, posent la question du regard genré des professionnels et de leur réflexivité quant aux stéréotypes et normes de genre. Comment se constitue ce regard, dans quelle mesure est-il informé par des stéréotypes extérieurs à l'institution ou par des prescriptions directes ? 
Quels sont les éléments qui, dans ce regard genré, discriminent l'appréhension des dossiers des filles et des garçons ? Il paraît important, pour conclure, d'approfondir et de préciser les hypothèses tirées de cette enquête de terrain.

\section{Intimité des adolescents et regard genré des professionnels de la justice des mineurs : hypothèses et éléments de conclusion}

Les éléments empiriques présentés plus haut donnent lieu à deux hypothèses principales et corrélées : celle d'un regard professionnel orienté par une grille de lecture genrée des situations et par des attentes de genre spécifique, et celle du rôle prépondérant du traitement institutionnel de l'intimité dans la production et reproduction de l'ordre de genre au tribunal pour enfants.

La première hypothèse invite à voir dans les éducateurs du service d'investigation, l'UEAT, des producteurs de normes genrées. Les écrits étudiés et les propos des éducateurs montrent que ces normes renvoient à un univers de représentation lié aux attentes de genre ${ }^{\mathrm{xiv}}$ les plus communes. Par attentes de genre, on entend ici le fait d'opérer une distinction dans l'appréhension institutionnelle des mineurs, au sens où les adolescentes seraient plus enclines à une mise en danger d'elles-mêmes, justifiant ainsi un besoin de protection par l'institution, et où les garçons seraient davantage concernés par la délinquance, impliquant de la part des professionnels rappel à l'ordre et répression (la délinquance consisterait ainsi essentiellement en une pratique de socialisation juvénile masculine Isabelle Coutant parle d'ailleurs de la délinquance comme d'un « fait social masculin »-, Coutant, 2007 : 113). On peut utilement se référer à la définition du genre proposée par Eleni Varikas, lorsqu'elle écrit que « le genre n'est pas seulement un principe d'ordre, fondé sur une division sociale des tâches et des fonctions différenciées ; c'est également une grille de lecture, une manière de penser le monde et le politique, à travers le prisme de la différence des sexes »(Varikas 2006 : 17). Dans le cas des écrits produits par les éducateurs, l'interprétation des situations paraît en effet renvoyer à la différence des sexes comme horizon général de l'analyse. S'il s'agit d'un horizon général et non de principes spécifiques, c'est que les enjeux liés à la différence des sexes ne sont jamais explicites dans les propos des éducateurs. On peut supposer, par exemple, que leur appréhension des dossiers de garçons est guidée par une association entre socialisation juvénile masculine et violence, alors que ceux des filles renvoient davantage à une étiologie psychologique, à une violence contre soi, nécessitant et justifiant un surcroit de protection. Tout se passe comme si les priorités éducatives n'étaient pas les mêmes selon le sexe des mineurs. La scolarité ou la formation paraissent être centrales dans l'analyse des situations des garçons, tout comme leur inscription dans une dynamique de responsabilisation (professionnelle, personnelle, sociale), alors que ces éléments semblent secondaires dans l'appréhension des conduites déviantes des filles, à propos desquelles on met davantage l'accent 
sur les problématiques de souffrance psychique, de relation familiale difficile ou de relation intime (sexuelle, ou conjugale pour les plus âgées).

Ce dernier point amène à une seconde hypothèse concernant les modalités de la différenciation du traitement judiciaire selon le genre. Il s'agit de l'hypothèse selon laquelle la question de l'intimité (et par extension de la sexualité), exceptionnellement abordée dans les dossiers des garçons, constitue au contraire une entrée privilégiée pour l'analyse des situations des adolescentes, discriminant fortement la nature de l'approche institutionnelle des dossiers selon le genre. S'il semble malaisé de distinguer, à partir des matériaux recueillis, la part de la présentation de soi des mineurs dans la prise en compte par les professionnels de problématiques liées à l'intimité, les dossiers n'en révèlent pas moins un biais genré évident que le travail sur les dossiers judiciaires dans leur ensemble (au-delà des seuls écrits éducatifs) m'a permis de mettre au jour. J'ai analysé différentes pièces versées aux dossiers judiciaires pour plus de deux cents dossiers récemment archivés. La lecture de ces dossiers donne à l'enquêteur l'impression d'une gêne des éducateurs face aux situations des jeunes garçons: les difficultés psychologiques et l'intimité de ces adolescents semblent relativement inaccessibles aux éducateurs, quel que soit d'ailleurs le sexe de ces professionnels. Par opposition, il n'est pas rare de lire dans des procès-verbaux policiers une référence à la sexualité d'une jeune fille, quand bien même il ne s'agit pas d'une affaire à dimension sexuelle. Je m'en tiendrai ici à deux exemples, qui témoignent de manière frappante de cette différence dans l'appréhension institutionnelle de l'intimité des adolescents, et ce d'autant plus qu'ils concernent précisément des affaires sexuelles - dans lesquelles on s'attend à voir l'intimité des justiciables inévitablement questionnée.

Sofiane est jugé tardivement dans une affaire ayant déjà fait l'objet de diverses procédures par le passé, et dont l'aboutissement est une poursuite pénale pour atteinte à la vie privée. Âgé de 17 ans au moment des faits qui lui sont reprochés, Sofiane entretenait une relation avec une jeune fille de 14 ans, avec qui il dit avoir eu plusieurs relations sexuelles. Même si divers éléments semblent faire douter le juge, il semblerait que la jeune fille ait été consentante au début de sa relation avec Sofiane. Peu de temps après ses relations sexuelles avec Sofiane, la jeune fille apprenait à ses dépens qu'une vidéo circulait à son propos, la montrant en train d'avoir une relation sexuelle avec ce garçon dans une cage d'escalier. La police n'est pas parvenue à récupérer la dite vidéo, mais le jeune homme finit par avouer avoir filmé l'adolescente sans son consentement. De nombreux éléments du dossier judiciaire renseignent sur le vécu de l'adolescente, décrite dans un rapport de l'assistante sociale du Collège comme «détruite» et «se sentant sale». Un rapport d'expertise psychologique la décrit comme «borderline » et « influençable », et un procès-verbal établi par la Police lors de son audition précise qu' « elle ne parvient pas à dire non aux garçons » et qu'elle est traitée de «pute » dans son quartier. Enfin, l'audition de son père révèle que ce dernier était au courant et qu'il désapprouvait les relations sexuelles de sa fille avec Sofiane. Dans ce dossier - une procédure pénale à l'encontre d'un jeune garçon -, on ne compte pas moins de trois documents faisant explicitement référence à la sexualité de 
la jeune fille, que l'on interroge sur son intimité et que l'on confronte aux dires de son père et de l'accusé à son égard. À l'inverse, la sexualité de Sofiane est littéralement absente du dossier, mis à part dans ses dimensions juridiques et "techniques » (les rapports sexuels étaient-ils consentis ? Étaient-ils filmés ?). Aucune expertise psychologique ou psychiatrique ne sera demandée par le juge. Le plus étonnant dans cette affaire est sans doute de voir l'attitude de la victime mise en cause pour sa gestion maladroite de son intimité, alors que le jeune garçon demeure protégé de toute intrusion institutionnelle dans sa sexualité.

Un autre garçon jugé au même moment par le tribunal pour enfants, Jason, semble davantage inscrit dans une trajectoire de déviance. Son dossier fait mention du repérage de cette " grande violence » par une assistante sociale dès son entrée au collège. Une investigation judiciaire en assistance éducative révélera que cette violence résulte d'un climat familial décrit comme "pathogène ». Avec une mère handicapée et un père possiblement violent (il ne sera toutefois pas inquiété, en l'absence de preuves suffisantes), Jason débute son adolescence de manière chaotique. Suite à plusieurs signalements, il sera placé en foyer dans l'Essonne, où il agressera à plusieurs reprises des éducateurs. À 14 ans, il est suivi par le juge des enfants en assistance éducative et au pénal, poursuivi pour vol et dégradation dans son foyer d'accueil. De plus, son dossier en assistance éducative révèle qu'il a échappé à une poursuite pour agression sexuelle sur une jeune fille du foyer, poursuite abandonnée pour manque de preuve - la jeune fille l'accuse toutefois d'attouchements sexuels, lesquels auraient eu lieu dans la chambre de Jason au foyer. Là encore, aucune expertise n'a été demandée. On trouve seulement dans le dossier ouvert en assistance éducative une évaluation psychologique de Jason datant du début de la procédure deux ans plus tôt. Cette évaluation concluait à «l'absence de trouble majeur » en se focalisant sur les difficultés existant entre le garçon et son père. Lorsque qu'un rapport émanant du foyer de placement du jeune homme tente d'apporter une explication à son comportement, la sexualité demeure dans l'ombre pour laisser place à une analyse renvoyant aux rapports du jeune homme à l'autorité (celle du père) et à l'altérité (« les femmes ») :

«De manière générale, Jason est agité, hyperactif, il ne tient pas en place. Au foyer, il fugue tout le temps. Il rend visite à ses grands-parents ou à son père. Dès son arrivée en décembre 2010, Jason fugue du foyer durant plusieurs jours. Il réapparaît brièvement sur le pavillon. À son retour, il raconte qu'il est allé chez ses grands-parents pour se laver et se changer entièrement, ce qu'il refuse de faire au foyer. Jason provoquerait la violence des hommes car il attendrait la réponse du père. Vis-à-vis des femmes, il les provoquerait, rechercherait leur affection pour ensuite, dans une ambivalence, les repousser en leur proférant des paroles blessantes $»^{\mathrm{xv}}$.

On remarque, à travers ces deux exemples, la manière dont l'intimité renforce l'ordre de genre, en allant jusqu'à faire porter une grande partie de l'attention des professionnels sur les victimes - des adolescentes que les professionnels n'hésitent pas à pathologiser dans le courant de la procédure, en laissant dans l'ombre des jeunes garçons à propos desquels on peut soupçonner l'existence de difficultés d'ordre sexuel et affectif. Cette absence de mise en lumière des difficultés intimes des 
garçons renforce indéniablement l'invisibilité et la neutralité classiquement attachée à l'identité masculine dans les rapports de pouvoir, au sens où les ressorts intimes de ces rapports sociaux et la manière dont ils sont vécus par les hommes demeurent généralement non-questionnés ${ }^{\mathrm{xvi}}$.

On peut ainsi avancer que les éducateurs, magistrats et autres acteurs de la chaîne pénale, font la différence des sexes et véhiculent des normes de genre dans leur activité (qualifications des situations ou des faits, orientation des dossiers, décisions de justice). La production institutionnelle du genre a ses régularités, mais elle ne semble pas répondre à un programme explicite de l'institution. À l'instar des street-level bureaucrats identifiés par Michael Lipsky, ces «travailleurs du service public qui interagissent directement avec les citoyens dans leur travail, et qui ont un pouvoir discrétionnaire substantiel dans l'exécution de leur travail» (Lipsky 2010 [1980] : 3, je traduis), les éducateurs de l'UEAT, et, suite à leurs recommandations, les magistrats, exercent un pouvoir qui dépasse les seules dimensions techniques (juridiques, éducatives) de leur activité. Ils produisent des jugements sur les familles, la jeunesse, ou expriment des attentes normatives face, par exemple, à ce que doit être la réinsertion d'une fille ou d'un garçon dans un contexte donné. On peut à cet égard faire le constat d'une persistance des conceptions « socio-éducatives » de la justice des mineurs, et ce alors même que les récentes réformes visaient à «techniciser» le travail éducatif et à rendre plus automatique le traitement des déviances (à l'instar des peines plancher, visant à garantir un niveau de pénalisation minimal pour les infracteurs).

Ce faisant, la prise en compte du genre dans l'étude qualitative de l'activité judiciaire contribue à une « ethnographie de l'État en actes » (Bouagga 2012), c'est-à-dire à une ethnographie qui prend pour point de départ des actes multiples, observables, parfois mesurables, pour les confronter ensuite à la figure de l'État comme structure idéologique et abstraite. On montre ainsi que derrière l'apparente neutralité de l'État comme deus absconditus (Bourdieu 2012 : 26), des logiques genrées sont à l'œuvre et participent à la reproduction, au sein même des institutions, des normes de genre les plus communes. Mais la neutralité de l'État pourrait également être questionnée à partir de la manière dont sont construites et régulées les politiques publiques du point de vue du genre. Il est à cet égard utile de rappeler le rôle de l'État dans l'organisation de politiques publiques genrées, car orientées différemment selon le sexe de leurs destinataires. Des recherches ont montré, par exemple, que les femmes font l'objet d'un contrôle parfois moins visible mais plus diffus dans leurs trajectoires de déviance, à l'instar du traitement pénal des « mauvaises mères » (Cardi 2007 : 15). Les parcours de ces mères condamnées à des peines de prison, le plus souvent suite à une accumulation de délits mineurs, révèlent qu'elles ont fait l'objet d'un contrôle sur la maternité et sur leurs conditions de vie (par les centres de Protection maternelle et infantile, la Protection judiciaire de la jeunesse, etc.) bien avant d'être condamnées pour des délits commis, pour la plupart, dans la continuité de leur expérience de stigmatisation sociale. Moins lourdement condamnées au pénal que les hommes, elles sont en revanche davantage contrôlées dans le cadre de politiques de contrôle des populations. Ainsi, la mise en évidence de biais de genre dans la justice pénale des mineurs doit nous inciter à porter un regard 
renouvelé sur les autres modalités du contrôle des déviances adolescentes (familiales, scolaires, médicales, etc.), car elles pourraient se révéler complémentaires du traitement pénal en tant que tel, et expliquer les mécanismes de transfert du traitement social des adolescentes délinquantes vers les institutions para-pénales.

\section{Bibliographie}

Aubusson de Cavarlay, Bruno. 2013. «Les mineurs mis en cause selon les statistiques de la police», Questions pénales, vol. XXVI, n² : 1-4.

Bouagga, Yasmine. 2012. "Le métier de conseiller d'insertion et de probation : dans les coulisses de l'État pénal ? », Sociologie du travail, vol. 3, n54 : 317-337.

Bourdieu, Pierre. 2012. Sur l'État. Cours au Collège de France 1989-1992. Paris, Seuil.

Cardi, Coline. 2007. «Le contrôle social réservé aux femmes : entre prison, justice et travail social», Déviance et société, vol. $31, \mathrm{n}^{\circ} 1: 3-23$.

Cardi, Coline. 2008. «Le féminin maternel ou la question du traitement pénal des femmes», Pouvoirs, ${ }^{\circ} 128: 75-86$.

Cassagnabère, Bernard. 1996. «Le mineur et la prison. Le Service éducatif auprès du Tribunal en première ligne », Revue pénitentiaire et de droit pénal, $\mathrm{n}^{\circ} 2: 159-182$.

Coutant, Isabelle. 2007. «Délinquance juvénile et rapport aux institutions socio-éducatives », in : Eckert, Henry, Faure, Sylvia. 2007. Les jeunes et l'agencement des sexes. Paris, La dispute : 113-129.

Delabruyère, Dominique, Haral, Cynthia. 2004. «L'activité des tribunaux pour enfants en 2003 », Infostat Justice, $\mathrm{n}^{\circ} 76: 1-4$.

Fassin, Éric. 2014. «Actualité des masculinités », in : Connell, Raewyn. Masculinités. Enjeux sociaux de l'hégémonie (sous la direction de Meoïn Hagège et Arthur Vuattoux). Paris, Amsterdam : 273-280.

Herpin, Nicolas. 1977. L'application de la loi. Deux poids, deux mesures. Paris, Seuil.

Hyde, Abbey et al. 2005. «The focus group method: Insights form group interviews on sexual health with adolescents », Social Science \& Medicine, vol. 61: 2588-2599.

Lipsky, Michael. 2010 (1980). Street-level Bureaucracy: Dilemmas of the Individual in Public Services. New-York, Sage Publications.

Ministère de la Justice. 2013. Les condamnations 2011, Paris, Ministère de la Justice. 
Ocqueteau, Frédéric. 2006. «Des femmes plus délinquantes, ou des regards policiers moins tolérants ? À propos des données de l'Observatoire national de la délinquance », Les cahiers de la sécurité, $\mathrm{n}^{\circ} 60: 35-40$.

Parini, Lorena. 2010. "Le concept de genre: constitution d'un champ d'analyse, controverses épistémologiques, linguistiques et politiques », Socio-logos, n5. URL: http://sociologos.revues.org/2468 (Consulté le 17/07/2014).

Revillard, Anne. 2013. "Administration», in: Achin, Catherine, Bereni, Laure (dir.). 2013. Dictionnaire genre \& science politique. Paris, Presses de Science-Po : 43-54.

Scherr, Mickael, Perron-Bailly, Etienne. 2012. «Nombres et profils des mis en cause pour crimes et délits non-routiers en 2011 », Observatoire national de la délinquance et des réponses pénales, Grand Angle, $\mathrm{n}^{\circ} 30: 1-98$.

Vanhamme, Françoise, Beyens, Kristel. 2007. «La recherche en sentencing: un survol contextualisé », Déviance et société, vol. 31, n 2 : 199-228.

Varikas, Eleni. 2006. Penser le sexe et le genre. Paris, PUF.

West, Candace, Zimmerman, Don, F. 1987. « Doing Gender », Gender and Society, vol. 1, n 2: 125151.

On utilisera ici fréquemment le terme « adolescentes », cette recherche concernant des mineurs de 13 à 18 ans (l'âge de 13 ans correspondant au seuil à partir duquel des sanctions éducatives au pénal peuvent être décidées par un juge des enfants). On trouvera également la notion de "délinquance des filles », correspondant aux termes du débat public sur la délinquance des mineurs, ainsi que des catégories propres aux institutions étudiées (« mineures », « femmes mineures », etc.).

Le terme sentencing renvoie à un domaine de recherche interdisciplinaire bien développé aux Etats-

Unis concernant les enjeux sociaux de la pénalité. Voir notamment Vanhamme, Beyens, 2007.

iii La chaîne pénale désigne, dans le langage de l'institution judiciaire, l'ensemble des étapes du processus pénal, à partir des « arrestations » ou « mises en cause » d'un individu par la Police ou la Gendarmerie jusqu'à la décision judiciaire et son application.

iv J'utiliserai ici le terme "genré » en tant que manière de désigner des enjeux renvoyant aux normes et principes de distinction basés sur une appréhension différentielle des sexes. Dans sa version la plus empirique, envisagé du point de vue des éducateurs, le concept de genre peut être rapporté à « la pratique qui consiste à organiser des conduites à la lumière de conceptions normatives des attitudes et activités appropriées selon chaque catégorie de sexe » (West, Zimmerman 1987 :127).

v L'« état 4001 » recense l'ensemble des crimes et délits transmis au Parquet par les services de Police et les unités de Gendarmerie

vi Résultat issu des données de l'ONDRP à partir du volume des mineures mises en cause en 2011 (33 949) parmi le total des mineurs (filles et garçons) mis en cause (196 497). L'année 2011 est prise comme point de référence car elle correspond à l'année de clôture de la plupart des dossiers judiciaires étudiés lors de l'enquête de terrain.

vii Données communiquées par la Sous-direction des statistiques et des études au ministère de la Justice à l'occasion de cette recherche. Les dernières données publiées à ce sujet datent de 2004 (Delabruyère, Haral 2004) et concernent l'année 2003.

viii L'enquête de terrain a notamment permis d'observer, sans pouvoir en rendre compte quantitativement, des situations où la composition pénale était utilisée à l'encontre d'adolescentes primo-délinquantes ne présentant pas un profil de déviance attendu, à l'instar d'adolescentes scolarisées au Lycée et vivant dans des familles aisées, pourtant mises en causes pour un trafic de drogue relativement important, et donnant donc habituellement lieu à des poursuites plus contraignantes.

ix Les focus groups sont des entretiens collectifs, préparés par l'enquêteur (notamment via une grille de questions ou, dans le cas présent, l'analyse d'un cas). Ils visent à « capturer les dynamiques interactionnelles de groupe et [à] les exploiter dans le but de comprendre un sujet» (Hyde et al. 2005 : 2588-2589). Il s'agit notamment de repérer, dans un groupe donné, les formes d'accord, de désaccord ou autres éléments d'interaction (humour, moqueries, etc.) qui émergent d'un collectif. Les focus groups permettent d'affiner la compréhension 
des dynamiques professionnelles et de faire émerger des questionnements qui n'étaient pas nécessairement présents au début de l'enquête.

Ordonnance n $45-174$ du 2 février 1945 relative à l'enfance délinquante (version consolidée du 7 août 2013), chapitre I, article 12

xi Circulaire d'orientation du 31 décembre 2010 relative à la mesure judiciaire d'investigation éducative, parue au Journal Officiel du 31 janvier 2011.

xii On désigne par "plafond de verre » le phénomène observé par les études féministes du travail, selon lequel «le taux de féminisation décroît à mesure que l'on progresse dans la hiérarchie des postes" (Revillard $2013: 51$ )

xiii Les noms et prénoms des éducateurs, comme ceux des mineurs, sont ici anonymisés.

xiv L'expression « attentes de genre», assez peu employée en français, est courante dans la littérature issue des gender studies (anglo-saxonnes). Comme l'écrit Lorena Parini, l'un des objectifs des études de genre est précisément de décrire, à partir des réalités étudiées, « un système de prescriptions et d'attentes sociales qui produisent de la différence et de la hiérarchie entre les sexes » (Parini, 2010).

xv Rapport d'un foyer éducatif transmis au juge des enfants dans le cadre d'une mesure en assistance éducative.

xvi Comme l'écrit Éric Fassin à propos du développement des men's studies à la suite des women's studies : «Pourquoi donc développer ensuite, en contrepoint, un champ nouveau de men's studies? C'est que les hommes, en tant que tels, s'avéraient paradoxalement invisibles. L'évidence de leur présence dans le tableau de la connaissance tenait à leur statut : c'était encore et toujours l'homme, implicitement universel ; mais qu'en était-il des hommes dans leur spécificité, soit une fois qu'on passait du singulier à la singularité ? (Fassin $2014: 273)$. 